\title{
Study on an automatic detection device for the enclosed turbine runner chamber based on real-time image acquisition
}

\author{
Xiangwei Yang, Yunlong Liu ${ }^{a}$, Wenbo Li, Dengke Li and Dingjie Shen \\ State Grid Hunan Electric Power Company Research Institute, Changsha 410007, China
}

\begin{abstract}
Due to the consideration of the existing testing technical problems for the key hidden metal components of the turbine runner chamber, such as the poor reliability and the larger detection blind spots under the downtime without opening the cover of the hydropower turbine runner chamber, an automatic detection device for the enclosed turbine runner chamber based on real-time image acquisition was designed. By using the permanent magnet wheel, the magnetic crawler which carries the real-time image acquisition device could complete the crawling work on the ferromagnetic surface of inclination angle of less than 55 degrees. Then the image acquisition device completed the real-time collection of the scene image of the enclosed turbine runner chamber. The positioning auxiliary device could be used to obtain the position of the magnetic crawler, and the steering and transmission device could be used to achieve the real-time image acquisition of 180 degree viewing angle through coordinated control of two motors. Then the defects or foreign matter fall into could be accurately positioning, in order to clean up and eliminate system error conveniently without opening the cover of the hydropower turbine runner chamber.
\end{abstract}

Keywords: real-time image acquisition; decompression panel; water board; automatic detection; enclosed turbine runner chamber.

\section{Introduction}

A plurality of hydropower plants at home and abroad have been occurred serious accidents, such as cover rubbing and damaged fault [1]. For example, at the Russia Sayano-Shushenskaya hydroelectric power station, the cover damage lead to the flooding plant serious accident that deprived more than 74 lives; at Ankang, Yantan, Danjiangkou and Panjiakou etc. hydropower plants, it has been frequent occurred the splitting cover and flooding plant accident [2].

The turbine cover of the hydropower plants in Hunan province also appeared abrasion. To prevent major accidents of the turbine, it is necessary to carry out the technology research of the fault detection and protection monitoring for the key components under the turbine cover. However, it is a time-consuming and labor-intensive work to open the cover of the turbine runner chamber. So the cover is just opened during A-type repair. When making B-type or C-type repair in hydropower plant, the endoscope is often used to extended into the chamber under the closed cover from the vacuum breaking valve hole above or the relief hole below to observe the key components such as the decompression panel and the water board in the enclosed turbine runner chamber [3]. Since the endoscope hose manual

\footnotetext{
a Corresponding author : 13874809964@163.com
} 
operation, the length and angle of the inserted probe cannot be controlled, it is difficult to determine the specific location of the inner chamber of the detection probe. Even if the defects or foreign matter is observed, it also cannot effectively guide the maintenance staff to repair. What's more, there is a big blind spot observation [4].

In this paper, an automatic detection device for the enclosed turbine runner chamber based on real-time image acquisition was designed. By using the permanent magnet wheel, the magnetic crawler which carries the real-time image acquisition device could complete the crawling work on the ferromagnetic surface of inclination angle of less than 55 degrees. Then the image acquisition device completed the real-time collection of the scene image of the enclosed turbine runner chamber. The positioning auxiliary device could be used to obtain the position of the magnetic crawler, and the steering and transmission device could be used to achieve the real-time image acquisition of 180 degree viewing angle through coordinated control of two motors. Then the defects or foreign matter fall into could be accurately positioning, in order to clean up and eliminate system error conveniently without opening the cover of the hydropower turbine runner chamber.

\section{Principle of automatic detection system}

The principle block diagram of the automatic detection system is shown in Fig. 1, which contains the computer control and display systems, the integrated module for data acquisition and processing, the magnetic crawler, the steering and transmission device, the positioning and distance measuring device, the image acquisition and illumination device and the DC power supply.

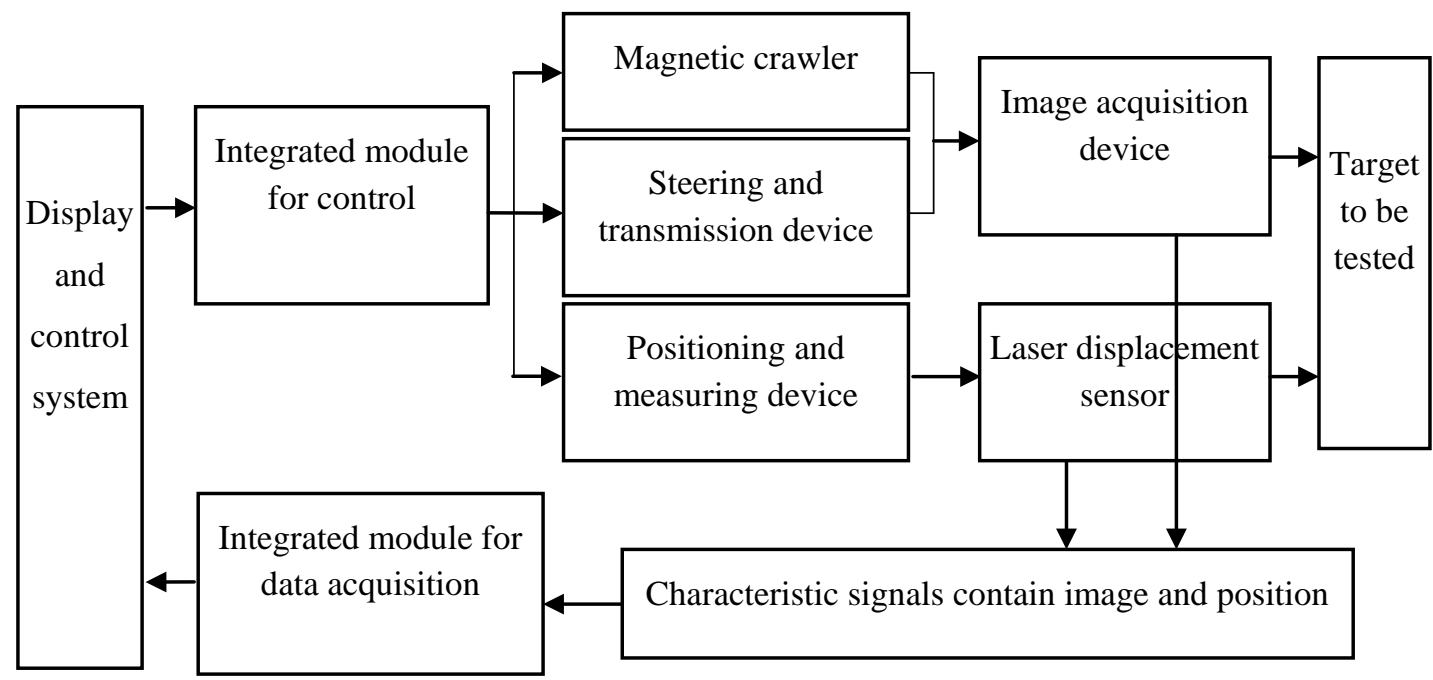

Figure 1. The principle block diagram of the automatic detection system

By using the permanent magnet wheel, the magnetic crawler which carries the real-time image acquisition device could complete the crawling work on the ferromagnetic surface of inclination angle of less than 55 degrees. Then the image acquisition device completed the real-time collection of the scene image of the enclosed turbine runner chamber. The positioning auxiliary device could be used to obtain the position of the magnetic crawler, and the steering and transmission device could be used to achieve the real-time image acquisition of 180 degree viewing angle through coordinated control of two motors. Then the defects or foreign matter fall into could be accurately positioning, in order to clean up and eliminate system error conveniently without opening the cover of the hydropower turbine runner chamber. 


\section{Design of the magnetic crawler}

During the whole detection system, the magnetic crawler is one of the most important components, whose stability and reliability have direct influence on the scope and detection performance of the system. The schematic diagram of the magnetic crawler and the transmission device is shown in Fig. 2.

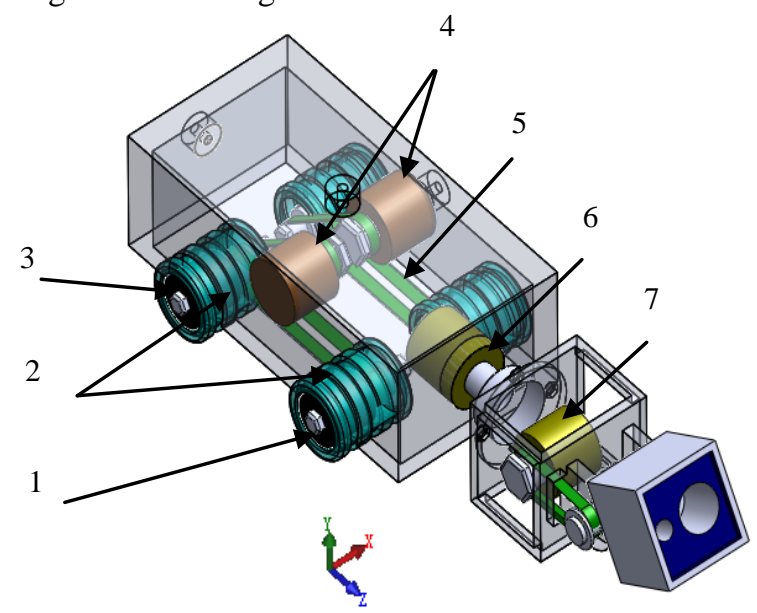

1- front wheels(driven wheels), 2- wear-resistant rubber wheel, 3- rear wheels(drive wheel), 4- drive motor, 5- belt, 6- motor around z-axis, 7- motor around x-axis.

Figure 2. Schematic diagram of magnetic crawler and transmission device

The magnetic crawler contains the front wheels (driven wheels), the rear wheels (driving wheel), the drive motor, the belt and the wear-resistant rubber wheel. All the wheels are made by the permanent magnet, and the magnetic crawler which carries the real-time image acquisition device could complete the crawling work on the ferromagnetic surface of inclination angle of less than 55 degrees.

\section{Design of the control system}

The flow chat of the detection control system is shown in Fig. 3. The detection control system contains the motor control system and the data acquisition and processing system, which could realize the coordinated control of the motors and complete interactive communication with data acquisition and processing module. The detection control system contains 4 motors, and a half of them are used to control the motion of the magnetic crawler, and the others are used to achieve the perspective control of the image acquisition and illumination device.

\subsection{Motion control}

The magnetic crawler has 2 driving wheels (or rear wheels) and 2 driven wheels (or front wheels). The driving wheel and the driven wheel on the same side are connected with the belt and the 2 driving wheels are controlled by 2 motors respectively. Through coordinated control of the 2 motors, it could be realized to control the magnetic crawler to forward, backward and steering.

\subsection{Viewing angle control}

The steering and transmission device contains 2 motors. One of them is used to achieve the 360 degree rotation around the $\mathrm{z}$-axis direction, and the other is used to achieve the 180 degree rotation around the $\mathrm{x}$-axis direction. It can be realized to achieve the real-time image acquisition of 180 degree viewing angle through coordinated control of two motors. 


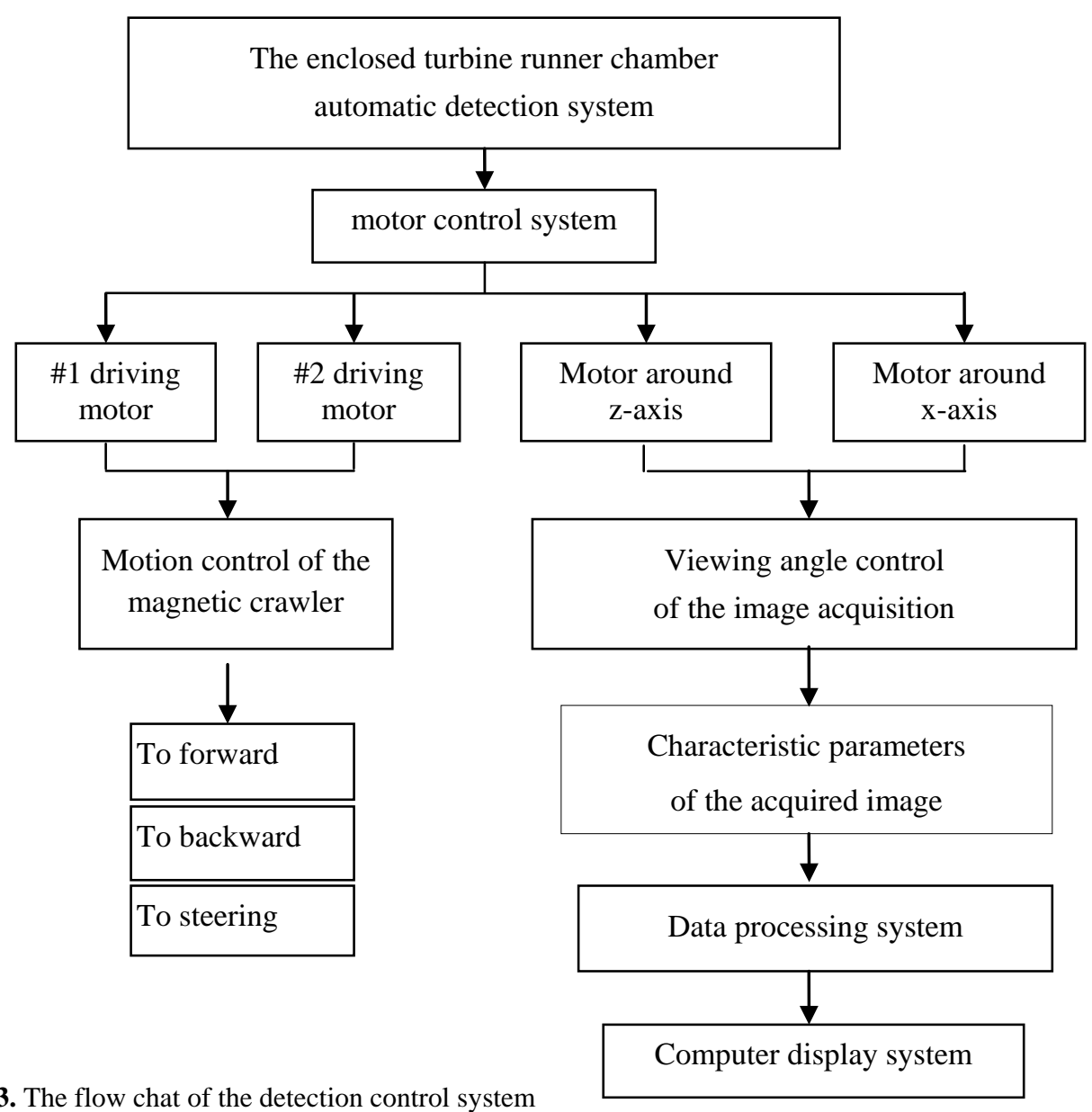

Figure 3. The flow chat of the detection control system

\subsection{Display control}

By using the positioning and distance measuring device, the characteristic parameters which contain the real-time position coordinates of the magnetic crawler and the acquired image could be obtained. Then the observation point and the position of the image could be calibrated and be display in the computer system.

\section{Application test}

By using the designed automatic detection device in Zhexi hydropower plants of Hunan province, it has been proved that the defects or foreign matter fall into could be cleaned up conveniently without opening the cover of the enclosed hydropower turbine runner chamber. It would be a useful method to the existing testing technical problems for the key hidden metal components of the turbine runner chamber. 


\section{Summary}

According to the technical problems of the existing testing methods for the key hidden metal components (such as the decompression panel and the water board) of the turbine runner chamber, such as the poor reliability, the inaccurate locating and the larger detection blind spots of the detection device, under the downtime without opening the cover of the hydropower turbine runner chamber, an automatic detection device for the enclosed turbine runner chamber based on real-time image acquisition was designed. By using the permanent magnet wheel, the magnetic crawler which carries the real-time image acquisition device could complete the crawling work on the ferromagnetic surface of inclination angle of less than 55 degrees. Then the image acquisition device completed the real-time collection of the scene image of the enclosed turbine runner chamber. The positioning auxiliary device could be used to obtain the position of the magnetic crawler, and the steering and transmission device could be used to achieve the real-time image acquisition of 180 degree viewing angle through coordinated control of two motors.

The field application test showed that the designed automatic detection device could clean up the defects or foreign matter conveniently without opening the cover of the enclosed hydropower turbine runner chamber. It is a useful method to the existing testing technical problems for the key hidden metal components of the turbine runner chamber, such as the poor reliability and the larger detection blind spots. It has important practical value to realize the automatic detection of the enclosed turbine runner chamber, to ensure the safety and reliability of operation.

\section{Acknowledgement}

This research was financially supported by the State Grid Hunan Electric Power Company (NO. 5216A514004A).

\section{References}

1. XIE Jianjun and LONG Jianming. Research progress of wear, Protection and control technology of domestic turbine, China Rural Water and Hydropower. 6(2007) 137-139.

2. TANG Hua. Rebirth of Russia Sayano-Shushenskaya hydroelectric power station, Energy. 12(2014)20-23.

3. CHEN Songyuan and TONG Xiaozhong. Vibration characteristics, analysis and diagnosis for rubbing between shaft/rotor and starts of turbine-generator in the field, Journal of Chinese Society of Power Engineering. 22 (2002)2020-2024.

4. ZHANG Guoshang, GAO Yimin, XING Jiandong, et al. Research on Erosion Damage of Typical Materials Used in Hydraulic Turbine, Foundry. 52(2003)932-935. 